\title{
The estimated risk of SARS-CoV- 2 infection via cornea transplant in Canada
}

\author{
Sheila F. O'Brien (iD) - Antoine Lewin (1D) Qi-Long Yi • Graeme Dowling • \\ Etienne Fissette $\cdot$ Steven J. Drews
}

Received: 29 October 2020/Accepted: 13 September 2021 / Published online: 30 September 2021

(C) The Author(s), under exclusive licence to Springer Nature B.V. 2021

\begin{abstract}
In late 2019 the respiratory illness, Corona Virus Disease-19 caused by the SARS-CoV-2 virus emerged in China and quickly spread to other countries. The primary mode of transmission is person-toperson via respiratory droplets. SARS-CoV-2 has been identified in conjunctiva. Transmission by cornea transplant has not been reported but is theoretically possible. We aimed to estimate the possible risk of transmission in Canada via cornea transplant during
\end{abstract}

S. F. O’Brien $(\bowtie) \cdot$ Q.-L. Yi

Epidemiology and Surveillance, Canadian Blood

Services, Ottawa, ON, Canada

e-mail: sheila.obrien@blood.ca

Q.-L. Yi

e-mail: qilong.yi@blood.ca

S. F. O'Brien · Q.-L. Yi

School of Epidemiology and Public Health, University of Ottawa, Ottawa, ON, Canada

\section{A. Lewin}

Medical Affairs and Innovation, Héma-Québec,

Saint-Laurent, QC, Canada

e-mail: antoine.lewin@hema-quebec.qc.ca

\section{A. Lewin}

Faculty of Medicine and Health Sciences, University of Sherbrooke, Sherbrooke, QC, Canada

G. Dowling

Comprehensive Tissue Centre, Alberta Health Services,

Edmonton Alberta, Canada

e-mail: graeme.dowling@albertahealthservices.ca the first wave of the pandemic, and the potential risk reduction from testing decedents. We constructed a deterministic model in which the risk of transmission was estimated as the product of three proportions: decedents with SARS-CoV-2 infection, corneas that are NAT positive, and NAT positive corneas presumed to transmit. Risk was estimated according to 3 scenarios: most likely, optimistic and pessimistic. At the peak of the first wave of the pandemic risk was

\author{
G. Dowling \\ Trillium Gift of Life Network, Toronto, \\ ON, Canada \\ E. Fissette \\ Medical Affairs and Innovation, Héma-Québec, Québec, \\ QC, Canada \\ e-mail: etienne.fissette@hema-quebec.qc.ca
}

\section{S. J. Drews}

Microbiology, Canadian Blood Services, Edmonton,

$\mathrm{AB}$, Canada

e-mail: steven.drews@blood.ca

\section{S. J. Drews}

Department of Laboratory Medicine and Pathology, University of Alberta, Edmonton, AB, Canada 
estimated to be 1 in 63,031 cornea transplants in Canada but could be as low as 1 in 175,821 or as high as 1 in 10,129 . It would take 16 years at the peak infection of the first wave of the pandemic to observe 1 transmission. Testing would reduce the risk of 1 in 63,031 to 1 in 210,104 assuming $70 \%$ test sensitivity. The theoretical risk of SARS-CoV-2 transmission by cornea transplant is extremely low and decedent testing is unlikely to be beneficial.

Keywords Cornea transplant · COVID-19 - SARSCoV-2 $\cdot$ Risk $\cdot$ Canada

\section{Introduction}

Cornea transplantation is one of the most common transplantation procedures, improving vision, reducing pain and increasing quality of life. In Canada, nearly 5000 cornea transplantations are performed each year, of which over 4000 are domestically sourced (Canadian Eye and Tissue Data Committee 2016). Viable corneas for transplantation are maintained in hypothermic preservation solution for up to 14 days, hence regular harvesting from deceased donors is essential to meet transplantation demand.

In late 2019, a novel virus, SARS-CoV-2, which primarily causes respiratory symptoms (SARS-CoV2, COVID-19), was identified in Wuhan, China (World Health Organization 2020), and quickly spread to other countries. By March 2020, cases were being identified in Canada. In March, an urgent teleconference meeting of the Canadian Eye and Tissue Donation Community was held, bringing together eye and tissue donation and bank leaders from across the country. Participants included administrative, medical and quality directors from banks, donation organizations, regulatory and professional associations. It was (and still is) unclear whether SARS-CoV2 can be transmitted by tissues from an infected donor to a recipient; however, at this time there is insufficient evidence to refute this possibility (Ho et al 2020). One of the urgent issues identified by the team was to estimate the risk of SARS-CoV-2 transmission via tissue transplantation, assuming that transmission is possible. Compared to most banked tissues, corneas require less processing between the harvesting and storage steps and might thus be more susceptible to disease transmission than other transplants. Moreover, reports suggest that the eye is a possible route of entry for SARS-CoV-2 (Ho et al 2020), and that some cells from ocular tissues might be permissive for viral replication. Given that more extensive processing is likely to reduce tissue contamination by SARS-CoV2 , the cornea was chosen as worst-case scenario for estimating the risk of SARS-CoV-2 transmission by tissue transplantation.

In Canada, eye and tissue banks have added screening questions to identify and exclude donors potentially infected or exposed to SARS-CoV-2. Testing tissue donors for SARS-CoV-2 by nasopharyngeal and oropharyngeal nucleic acid amplification testing (NAAT) or serological testing (when available) is performed at the discretion of the medical director. The American Association of Tissue Banks (2020) has reported that the Food and Drug Administration (FDA 2020) does not recommend testing of asymptomatic donors. Recommendations from the Eye Bank Association of America (EBAA) and the Global Alliance of Eye Bank Associations (GAEBA) are in line with those of the FDA (Desautels et al. 2020). Given that regulatory bodies currently do not require testing of tissue donors for SARS-CoV-2 infection, yet a theoretical risk of transmission by tissue transplantation still exists., We have developed and tested a mathematical model aimed at quantifying this risk.

\section{Methods}

The risk of a corneal transplant transmitting SARSCoV-2 without testing was estimated from the product of three terms:

Risk $_{C T}=P_{\text {Unidentified }} \times P_{\text {NATpos }} \times P_{\text {Transmit }}$

The proportion of the general population with asymptomatic or mild infection that clinicians would not be aware of ( $\left.\boldsymbol{P}_{\text {Unidentified }}\right)$ was estimated from the number of unresolved cases and adjusted for the proportion who would be in the pre-symptomatic period, thus not included in the reported cases (see Table 1). The proportion of patients that would have SARS-CoV-2 NAT-positive conjunctival tissue $\left(\boldsymbol{P}_{\text {NAT pos }}\right)$ was estimated from a meta-analysis of studies in which patients with known SARS-CoV-2 infection and with conjunctivitis had eye exudate or tears tested for SARS-CoV-2 nucleic acid (See 
Table 1 Parameters for most likely scenario

\begin{tabular}{|c|c|c|c|}
\hline Parameter & Approach & $\begin{array}{l}\text { Parameter } \\
\text { value }\end{array}$ & Data sources \\
\hline $\begin{array}{l}\text { Prevalence of active COVID- } \\
19 \text { infection }\end{array}$ & 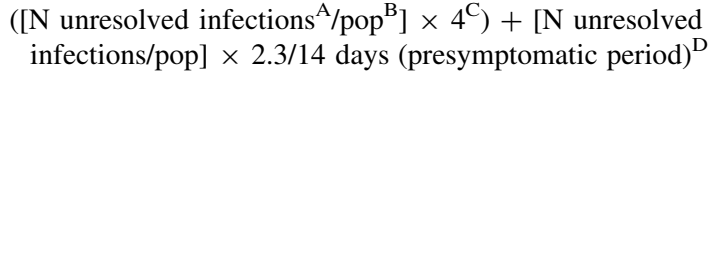 & $\begin{array}{l}\text { Varies by } \\
\text { date and } \\
\text { region }\end{array}$ & $\begin{array}{l}\text { A: Public Health } \\
\text { Agency of Canada } \\
\text { (2020b) } \\
\text { B: Statistics Canada } \\
\quad(2020) \\
\text { C: Tuite et al (2020) } \\
\text { D: He et al (2020b) }\end{array}$ \\
\hline $\begin{array}{l}\text { Probability of virus present in } \\
\text { the tissue if donor is infected }\end{array}$ & $\begin{array}{l}\text { Incidence of conjunctival congestion in COVID-19 } \\
\text { positive patients }\end{array}$ & $\begin{array}{l}1.95 \% \\
(\mathrm{CI} \\
0.74-4.1)\end{array}$ & Sarma et al (2020) \\
\hline Probability of transmission & & $50 \%$ & Assumption \\
\hline $\begin{array}{l}\text { Sensitivity of nasopharyngeal } \\
\text { swab testing }\end{array}$ & NAT vs CT, 1-sensitivity & $30 \%$ & Fang et al (2020) \\
\hline
\end{tabular}

Table 1). In the absence of high-quality data, the proportion of corneas from decedents with NATpositive eye exudate or tears that would transmit $\left(\boldsymbol{P}_{\text {Transmit }}\right)$ was theoretical and assumed to be $50 \%$.

Given the uncertainty in the values of some parameters, the risk was estimated according to three scenarios: (1) a "most likely" scenario, which we considered to reflect the actual risk, given the current state of knowledge, albeit with an appropriate level of caution (2) an "optimistic" scenario, in which parameter values were adjusted so as to attenuate the risk; and (3) a "pessimistic" scenario, in which parameters values were adjusted according to a "worst-case" scenario (see Table 2 for details). The proportion of infected corneas that would transmit SARS-CoV-2 if transplanted was also varied from the assumed $50 \%$ to $25 \%$ and $10 \%$, to compare the impact of this parameter adjustment. In order to estimate the risk associated with hypothetical decedent testing, the estimated risk was multiplied by (1-(sensitivity of current assays)), with sensitivity estimated at 70\% (Fang et al. 2020). However, given the uncertainty regarding assay sensitivity on cadaveric decedent samples, especially as mucosal specimen sampling might be impacted by post-mortem drying, parameter values of $50 \%$ and $80 \%$ were also tested.

This analysis makes a number of assumptions, the most important being:

1. It is assumed that SARS-CoV-2 can be transmitted via infected corneas.

2. Deceased donors with known SARS-CoV-2 infection would not be selected for cornea harvesting, thus risk estimates are solely based on

Table 2 Parameters for optimistic and pessimistic scenarios

\begin{tabular}{ll}
\hline Optimistic scenario & \\
Prevalence of active infections & Assumed to be 3 times the reported cases, rather than 4 for most likely scenario \\
Probability of virus in tissue if infected & $0.74 \%$ (lower end of confidence interval) \\
Probability of transmission & $50 \%$ \\
\hline Pessimistic scenario & \\
Prevalence of active infection & Assumed to be 3 times the reported cases, with a pre-symptomatic period of 3 days \\
Probability of virus in tissue if infected & $4.1 \%$ (upper end of confidence interval) \\
Probability of transmission & $50 \%$ \\
\hline
\end{tabular}


infectionsed donors that neither physicians nor families are aware of.

3. The proportion of SARS-CoV-2 positive decedents is equal to the proportion of people with SARS-CoV-2 that have not been identified by testing and is 4 times the proportion of individuals with known infections.

4. The probability of NAT-positive corneas can be estimated from studies of NAT testing in patients with eye symptoms, and those without eye symptoms are assumed to have NAT-negative corneas and thus cannot transmit the infection.

5. 50\% of decedents with NAT-positive corneas will transmit infection to recipients.

We note that our model assumes independence of the three terms. However, it is possible that they are not independent. For example, for decedents whose only symptom is conjunctivitis there may be less suspicion of infection, thus greater risk of transmission. Alternatively, a decedent with no symptoms may be less likely to have conjunctival viral particles, thus less risk of transmission. To address this possibility a sensitivity analysis was performed in which risk was estimated assuming potential negative correlations of $10 \%, 15 \%$ and $20 \%$ and potential positive correlations of $10 \%, 15 \%$ and $20 \%$ in the most likely scenario.

\section{Results}

The risk of SARS-CoV-2 infections varies according to the prevalence of active infections in the community and was very low in the early stage of the first phase of the pandemic from mid-March to mid-April 2020, and highest from mid-April to midJuly (Fig. 1). According to the most likely scenario, the risk at the peak of the first phase of the pandemic was about 1 transmission in 63,000 cornea transplants, but might have been as low as 1 in 175,000, or as high as 1 in 10,000, depending on whether an optimistic or a pessimistic scenario is tested (Table 3). These estimates assume that $50 \%$ of NAT-positive corneas would transmit the infection if transplanted. If the transmission rate was $25 \%$, the risk would be proportionally lower, at about 1 in 126,000 transplants, and for a transmission rate of only $10 \%$, the risk would be about 1 in 315,000 , according to the most likely scenario. As the peak active infection rate varied across provinces, so did the peak risk, with the highest risk in Quebec at about 1 in 1600 transplants, and the lowest in British Columbia at 1 in 415,000 transplants, according to the most likely scenario. The same scenario yields an average risk over that period of 1 in 63,061 across Canada. If about approximately 4000 cornea transplants are performed each year in Canada, it would take $\sim$ about 16 years for one infection to be transmitted by cornea transplantation. In sensitivity analyses that considered potential positive and negative correlation among the terms in the equation, varying the coefficient correlation from $-20 \%$ to + $20 \%$ had a modest impact on risk estimates (See Appendix Table 1).

If decedent testing was performed, assuming that $70 \%$ of infected donors would be identified, the risk would be proportionally reduced (Table 4). For

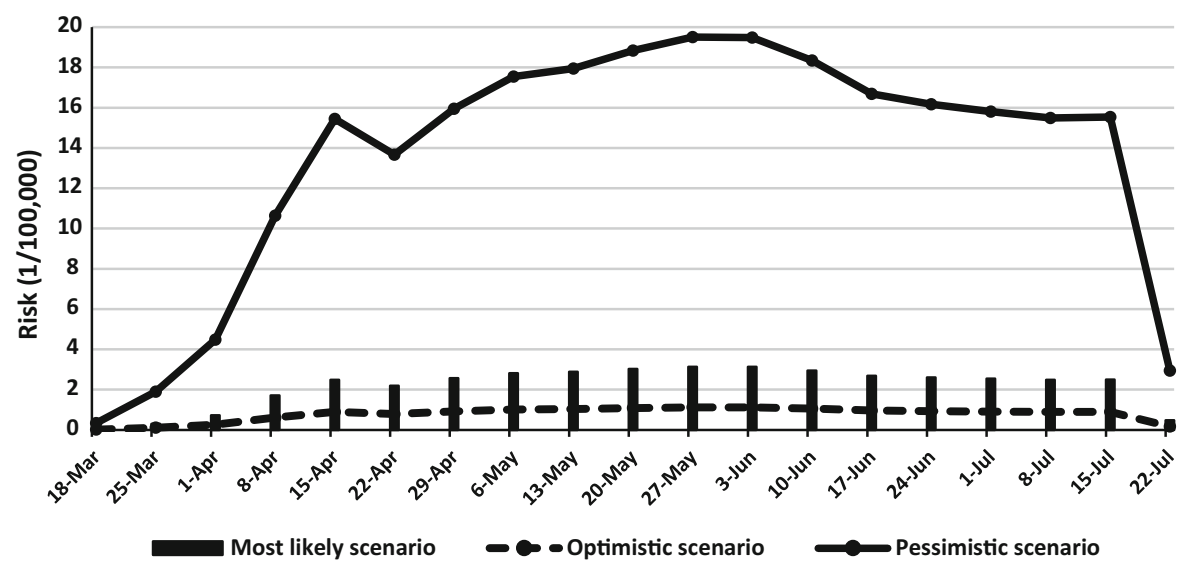

Fig. 1 Risk of cornea transplant transmission in Canada according to three scenarios, from March 18 to July 22, 2020 
Table 3 Risk of SARSCoV-2 transmission by cornea transplantation by province at their respective peak week in number of COVID-19 cases between March and June 2020

\begin{tabular}{llll}
\hline Province & \multicolumn{2}{l}{ Transmission risk } & \\
\cline { 2 - 4 } & Most likely scenario & Optimistic scenario & Pessimistic scenario \\
\hline British Columbia & 1 in 414,988 & 1 in $1,157,578$ & 1 in 66,685 \\
Alberta & 1 in 82,216 & 1 in 229,334 & 1 in 13,211 \\
Saskatchewan & 1 in 227,043 & 1 in 633,319 & 1 in 36,484 \\
Manitoba & 1 in 327,331 & 1 in 913,064 & 1 in 52,599 \\
Ontario & 1 in 94,671 & 1 in 264,078 & 1 in 15,213 \\
Quebec & 1 in 16,490 & 1 in 45,998 & 1 in 2,650 \\
Newfoundland and Labrador & 1 in 119,971 & 1 in 334,651 & 1 in 19,279 \\
New Brunswick & 1 in 388,115 & 1 in $1,082,618$ & 1 in 62,367 \\
Nova Scotia & 1 in 94,527 & 1 in 263,677 & 1 in 15,190 \\
Prince Edward Island & 1 in 354,139 & 1 in 987,844 & 1 in 56,908 \\
Canada & 1 in 63,031 & 1 in 175,821 & 1 in 10,129 \\
\hline
\end{tabular}

example, across Canada the peak risk would be reduced from about 1 in 63,000 transplants to about 1 in 210,000 transplants.

\section{Discussion}

Current evidence indicates that SARS-CoV-2 transmission via cornea transplant from an infected donor is merely a theoretical risk; yet, not a single case of transmission through this mode has been reported as of January 4, 2021 (FDA). However, it might take a while before undisputable cases of transmission are identified, for several reasons. First, it might be difficult to distinguish transplant-acquired and community-acquired infections. Second, transmission might not be ascertained, especially if the recipient remains asymptomatic. Finally, as suggested by our analysis, transmission by cornea transplant is likely a very rare event. Until such time as transmissibility is confirmed or refuted, it is prudent to assume that it may be transmissible.

Upon harvest, corneas are stored in Optisol-GS solution, which contains dextran and chondroitin sulphate to control stromal hydration, as well as vitamins and ATP precursors, but no antiviral agents. Storage is maintained at $2-8{ }^{\circ} \mathrm{C}$. Recent evidence suggests that SARS-CoV-2 infectivity is better maintained by cold temperatures $\left(4^{\circ} \mathrm{C}\right)$ than by storage at room temperature (Chin et al. 2020; Chan et al. 2020). Corneas may be stored for up to two weeks, but transplantation within one week from harvest is preferred. Thus, if SARS-CoV-2 survives the storage process, as suggested by some stability studies, a theoretical risk remains.
Our model accounted for the likelihood of a cornea transmitting SARS-CoV-2 from a deceased donor who was positive for the infection. We assumed that donors with known infections would be excluded from cornea harvesting due to because of screening criteria in place, although it is possible that symptomatic donors would not be identified by the physician, or that symptoms would not reported by the deceased donor's relatives. The proportion of deceased donors with known infections was based on public health data, if only one out of five infections would be identified, which would lead to unintended harvesting of corneas from infected donors. The proportion of infections identified by public health is dependsent on testing policies, which vary by jurisdiction and over time. Thus, it is possible that more people had symptoms than were tested (but would be identified by relatives in the case of a deceased tissue donor) and that the proportion of infected decedents with no known symptoms might be less lower than expected. Hence, it is possible that our estimate of the proportion of decedents with SARS-CoV-2 infection was not underestimated, and might have been rather conservative.

We have also assumed that the proportion of decedents who might have donated infected corneas could be estimated from reports of the proportion of infected patients with eye or conjunctival symptoms, and reports of the proportion of these patients that were positive for SARS-CoV-2 nucleic acid in the eye. Although we believe that this is the best strategy for quantifying these parameters at this time, there is inherent uncertainty in these estimations, for at least two reasons: (1) there are relatively few published data 


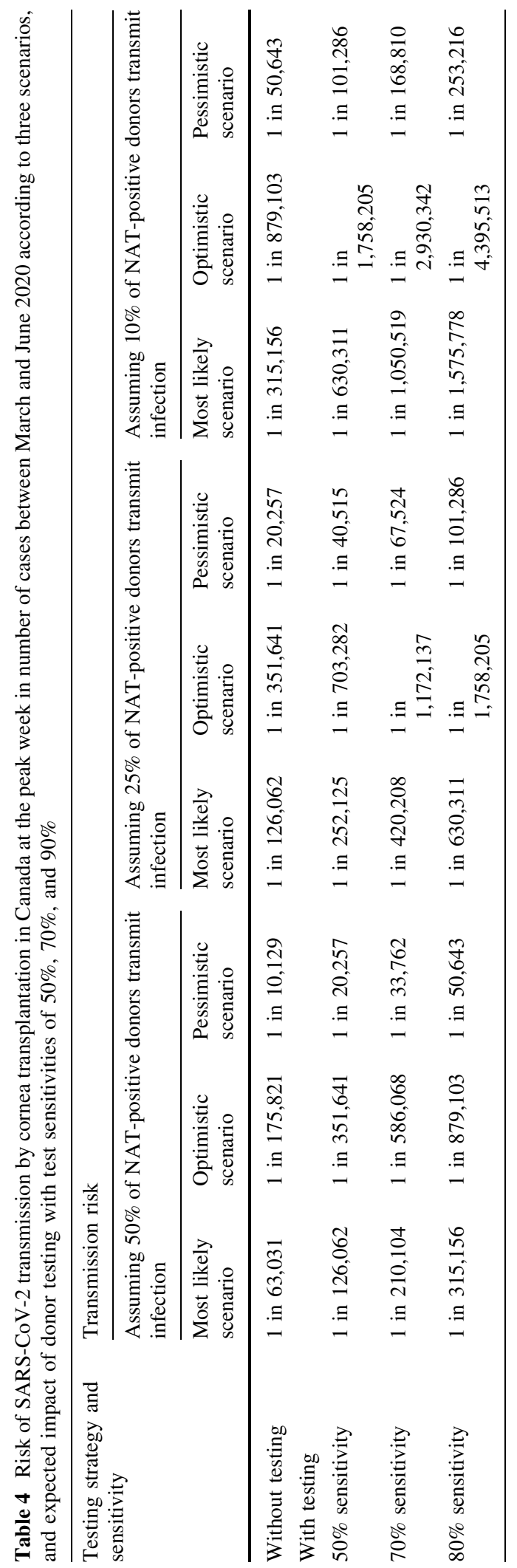


on SARS-CoV-2 infection in the eye; and (2) it is possible that corneas might be infected in the absence of conjunctivitis or exudate.

Our model also assumed that not all SARS-CoV2-contaminated corneas would transmit the infection. If indeed SARS-CoV-2—contaminated corneas could transmit the infection through transplantation, it is unlikely that transmission would occur for $100 \%$ of contaminated corneas. Given the paucity of available high-quality data, we assumed that $50 \%$ of transplanted SARS-CoV-2-positive corneas would establish an infection. As mentioned earlier, this figure may nonetheless represent an overestimate. In a recent study, SARS-CoV-2 RNA was detected in the corneal discs of 6 out of $11(55 \%)$ deceased patients with COVID-19 (Casagrande et al 2021). Of note, most patients included in this study had severe COVID-19 illness (i.e., 10 [91\%] died of COVID-19) and hence exhibited high viral loads. Despite that, none of these corneal discs demonstrated infectivity when incubated with a cell line. Establishing an infection depends on a range of factors including the infectious dose, the route of entry, and several host factors such as immune status, age, overall health, etc. Importantly, the cornea is not a vascular tissue; thus, it might be resistant to infection, and might act merely as a carrier of infectious virions. Therefore, the true risk of SARSCoV-2 transmission through cornea transplantation is probably even lower than the estimates presented in the current study.

Several observations point to the importance of maintaining a high level of vigilance regarding the risk of transmission of COVID-19 by cornea transplantation. First, numerous reports have documented the detection of SARS-CoV-2 RNA in ocular tissues (Xia et al. 2020; Li et al 2020; Zhou et al 2020; Wu et al 2020; Chen et al 2020a,b,c; Zhang et al 2020a,b,c; Sarma et al 2020; Colavita et al 2020). Second, one research team was able to demonstrate infectivity in ocular samples from COVID-19 patients by inoculation of cell lines that are permissive for SARS-CoV-2 infection (Colavita et al 2020); however, Seah et al (2020) and $\mathrm{Li}$ a et al. (2020) did not succeed in demonstrating infectivity of ocular samples in cell culture. Third, the ACE2 viral receptor and the TMPRSS2 serine protease required for cell entry are expressed in a variety of tissues and organs (Hamming et al 2004; Zou et al 2020; Muus et al. 2021; Sungnak et al 2020; Ziegler et al 2020; Li b et al. 2020; Fu et al
2020; Chen b et al. 2020; Hikmet et al 2020; Battagello et al 2020; Dong et al 2020; Singh et al 2020; Ortiz et al 2020) including ocular tissues (Sungnak et al 2020; Ma et al. 2020, Makovoz et al 2020, Hamashima et al 2020, Collin et al. 2021). Fourth, there is experimental evidence that SARS-CoV-2 can infect cells from human ocular tissues ex vivo (Makovoz et al 2020; Hui et al 2020). Additionally, Deng et al (2020) were able to infect a non-human primate via the conjunctival route. Collectively, these reports suggest that the eye is a possible route of entry for SARS-CoV2 , and that some cells from ocular tissues might be permissive for viral replication.

The contribution of the scientific literature in the evaluation of the risk of SARS-CoV-2 transmission by cornea transplantation should be assessed in a broader context. COVID-19 remains essentially a respiratory tract infection. Reports of COVID-19 - associated conjunctivitis are anecdotal, and generally involve more severe cases of COVID-19. To the best of our knowledge, there have is been very fewone reported cases suggesting that for which the eye wasmay have been the primary route of infection (Lu et al. 2020)., and Even in these very few cases, one cannot exclude the possibility that the virus entered the body via the respiratory system. Some authors have mentioned the anatomical connection between the nasolacrimal system and the upper respiratory airways as a possible route by which the virus could be carried from one compartment to the other (Belser et al 2013). The results of an experimental infection in a non-human primate animal model are consistent with this hypothesis (Deng et al 2020). Nonetheless, the available evidence strongly suggests that aside from anecdotal reports, virtually all COVID-19 cases result from primary infection of respiratory airways.

In the context of our risk assessment related to cornea transplantation, another remote possibility is that the virus could spread from a contaminated cornea transplant to other organs, including the lungs, via the lymphatic and circulatory systems, in a sort of retrograde movement from the basolateral side to the apical side of alveolar epithelial cells, which are prime targets for viral infection and tissue damage (Mason 2020). We believe that this hypothesis is unlikely, as it would imply that viral particles are able to cross endothelial cells, basement membranes, and interstitial spaces in the lung before finally reaching alveolar epithelial cells. Although the ACE2 receptor is 
expressed by diverse cells from several organs, the level of expression of ACE2 in a given cell type is not a reliable predictor of the susceptibility of that cell to SARS-CoV-2 infection (Oritz et al. 2020; Bojkova et al. 2021). Even in the worst-case scenario of a cornea transplant from an asymptomatic SARS-CoV2-positive donor, the available evidence and the results presented herein suggest that the risk of a transplant recipient developing a respiratory infection typical of COVID-19 appears to be very low.

Decedent testing prior to harvesting is not routinely performed in Canada, but it has been under consideration by various eye banks as a potential risk reduction strategy. A multifactorial approach considering clinical presentation, epidemiology/risk factors, laboratory tests and imaging has been suggested for the early identification of COVID-19 cases (Ai et al 2020; Qin et al 2020; Chen et al 2020c). Such a multifaceted strategy might reduce the chances of false-negative diagnoses. The sensitivity of NAT has been shown to fluctuate between studies, and may be impacted by several variables including: comparator or gold standard chosen (Zhang et al. 2020b,c), timing of sampling (Chen et al. 2020b), sample quality, anatomical site of sampling in the respiratory tract (LeBlanc et al. 2020; Vlek et al. 2021), assay used (van Kesteren et al. 2020) and clinical presentation. Compared to a COVID-19 reference standard, reported sensitivities range from 71 to $83 \%$ (Fang et al 2020; Long et al 2020; He et al 2020a), and a recent meta-analysis of seven studies suggests that the sensitivity of NAT on nasopharyngeal swab/aspirates and throat swab specimens was $73.3 \%$ (95\% CI 68.1-78.0\%) (Böger et al 2020). Nasopharyngeal samples obtained from decedents might have lower sensitivity due to drying of tissues prior to sampling. We have estimated the risk reduction provided by NAT performed on decedent samples using a sensitivity of $70 \%$, but we have also assessed the impact of sensitivities as low as $50 \%$ and as high as $80 \%$.

Our results suggest that the risk of SARS-CoV-2 transmission via cornea transplant is low in Canada, even when using relatively high prevalence data from the peak of the first wave of the pandemic. Risk varies as a function of the prevalence in the population but is systematically much lower than the risk of being infected by the conventional route of the respiratory tract. For example, assuming there are four undiagnosed cases for every diagnosed case in the general population, with a peak incidence of 2000 daily cases
(Public Health Agency of Canada 2020a), about 1 in 5,000 people would be expected to be infected at the peak infection rate, whereas the risk from a cornea transplant would be much lower, at about 1 in 63,000 as per our estimates. According to the pessimistic scenario, the risk would be about 1 in 10,000, if there are ten undiagnosed cases for every positive diagnosis. With that assumption, the proportion of the general population that would be infected is about 1 in 2000 .

Decedent testing would reduce the risk as a function of the sensitivity of the assay. For example, a risk of 1 in 63,000 at the peak of the pandemic could have been reduced to about 1 in 210,000 if testing had a sensitivity of $70 \%$. During non-pandemic times, about 100 cornea transplants are performed each week in Canada, and since most elective surgeries were cancelled during the peak of the pandemic, the number of transplants performed during that period must have been lower. Given that the SARS-CoV-2 transmission risk associated with cornea transplantation, based on our estimates, is already very low, the benefit of decedent testing appears to be negligible.

Authors' contribution All authors contributed to the study conception, design and parameter selection. Material preparation and analysis were performed by Sheila O'Brien, Antoine Lewin and Qi-Long Yi. The first draft of the manuscript was written by Sheila O'Brien and all authors commented on previous versions of the manuscript. All authors read and approved the final manuscript.

Funding No funding was received to assist with the preparation of this manuscript.

Data availability Data sharing is not applicable to this article as no datasets were generated or analysed during the current study.

Code availability Coding for these analyses available from Sheila O’Brien at sheila.obrien@blood.ca.

\section{Declarations}

Conflict of interest Steven J. Drews has acted as a content expert on respiratory viruses for Johnson and Johnson (Janssen). He also has acted as a content expert to Roche on Arboviruses. All other authors have no conflicts of interest to declare that are relevant to the content of this article.

Ethical approval Not applicable.

Consent for publication All authors consent to publication of this manuscript. 


\section{Appendix}

See Table 5

Table 5 Sensitivity analysis of potential non-independence: Risk of SARS-CoV-2 transmission by cornea transplantation in Canada at the peak week in number of cases between March and June 2020 and expected impact of donor testing with test sensitivities of $50 \%, 70 \%$, and $90 \%$

\begin{tabular}{|c|c|c|c|c|c|c|c|}
\hline \multirow{3}{*}{$\begin{array}{l}\text { Testing strategy and } \\
\text { sensitivity }\end{array}$} & \multicolumn{7}{|c|}{ Assuming $50 \%$ of NAT-positive donors transmit infection Transmission risk } \\
\hline & \multirow{2}{*}{$\begin{array}{l}\text { Most likely } \\
\text { scenario }\end{array}$} & \multicolumn{3}{|c|}{ Potential negative correlation $^{a}$} & \multicolumn{3}{|c|}{ Potential positive correlation $^{\mathrm{a}}$} \\
\hline & & $-10 \%$ & $-15 \%$ & $-20 \%$ & $+10 \%$ & $+15 \%$ & $+20 \%$ \\
\hline \multicolumn{8}{|l|}{ Most likely scenario } \\
\hline Without Testing & 1 in 63,031 & 1 in 70,035 & 1 in 74,154 & 1 in 78,789 & 1 in 57,301 & 1 in 54,810 & 1 in 52,526 \\
\hline \multicolumn{8}{|l|}{ With testing } \\
\hline $50 \%$ sensitivity & 1 in 126,062 & 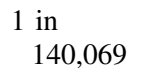 & 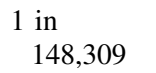 & 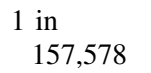 & 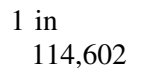 & 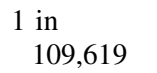 & 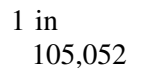 \\
\hline $70 \%$ sensitivity & 1 in 210,104 & 1 in 233,449 & 1 in 247,181 & 1 in & 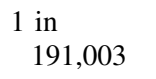 & 1 in 182,699 & 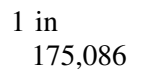 \\
\hline $80 \%$ sensitivity & 1 in 315,156 & 1 in 350,173 & 1 in 370,771 & 1 in 393,945 & 1 in 286,505 & 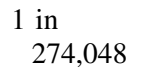 & 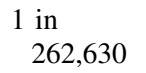 \\
\hline
\end{tabular}

${ }^{\mathrm{a}}$ Correlation between three risk component terms: $P_{\text {Unidentified, }} P_{\mathrm{NAT} \text { pos }}$ and $P_{\text {Transmit }}$

\section{References}

Ai T, Yang Z, Hou H et al (2020) Correlation of chest CT and RT-PCR testing for coronavirus disease 2019 (COVID-19) in China: a report of 1014 cases. Radiology 296:E32-40

American Association of Tissue Banks (AATB) (The) (2020) Bulletin 20-10-COVID-19 update. American Association of Tissue Banks (AATB) (The) 2020 https://www.aatb.org/ content/bulletin-20-10. Accessed 7 Oct 2020

Battagello DS, Dragunas G, Klein MO, Ayub ALP, Velloso FJ, Correa RG (2020) Unpuzzling COVID-19: tissue-related signaling pathways associated with SARS-CoV-2 infection and transmission. Clin Sci 134:2137-2160

Belser JA, Rota PA, Tumpey TM (2013) Ocular tropism of respiratory viruses. Microbiol Mol Biol Rev 77(1):144-156

Böger B, Fachi MM, Vilhena RO, Cobre AF, Tonin FS, Pontarolo R (2020) Systematic review with meta-analysis of the accuracy of diagnostic tests for COVID-19. Am J Infect Control 49:21-29

Bojkova D, McGreig JE, McLaughlin K-M et al (2021) SARSCoV-2 and SARS-CoV differ in their cell tropism and drug sensitivity profiles. Bioinformatics. https://doi.org/10. 1093/bioinformatics/btab094

Canadian Eye and Tissue Data Committee (2016) Donation and Transplantation-Canadian Tissue and Eye Banking Statistics 2016. Canadian Blood Services. https://profedu. blood.ca/sites/msi/files/2016_etdc_final_updated_report_ july_2018.pdf. Accessed 7 Oct 2020
Casagrande M, Spitzer MS, Puschel K et al (2021) Presence of SARS-CoV-2 RNA in the cornea of viremic patients with COVID-19. JAMA Ophthalmol 139:383-388

Chan K-H, Sridhar S, Zhang RR et al (2020) Factors affecting stability and infectivity of SARS-CoV-2. J Hosp Infect 106:226-231

Chen L-D, Li H, Ye Y-M et al (2020a) A COVID-19 patient with multiple negative results for PCR assays outside Wuhan, China: a case report. BMC Infect Dis 20:517

Chen L, Liu M, Zhang Z et al (2020b) Ocular manifestations of a hospitalised patient with confirmed 2019 novel coronavirus disease. Br J Ophthalmol 104:748-751

Chen Q-L, Li J-Q, Xiang Z-D, Lang Y, Guo G-J, Liu Z-H (2020c) Localization of cell receptor-related genes of SARS-CoV-2 in the kidney through single-cell transcriptome analysis. Kidney Dis 6:258-270

Chin AWH, Chu JTS, Perera MRA, et al (2020) Stability of SARS-CoV-2 in different environmental conditions. Lancet Microbe 1:e10

Colavita F, Lapa D, Carletti F et al (2020) SARS-CoV-2 isolation from ocular secretions of a patient with COVID-19 in Italy with prolonged viral RNA detection. Ann Intern Med 173:242-243

Collin J, Queen R, Zerti D et al (2021) Co-expression of SARSCoV-2 entry genes in the superficial adult human conjunctival, limbal and corneal epithelium suggests an additional route of entry via the ocular surface. Ocul Surf 19:190-200. https://doi.org/10.1016/j.jtos.2020.05.013 
Deng W, Bao L, Gao H et al (2020) Ocular conjunctival inoculation of SARS-CoV-2 can cause mild COVID-19 in rhesus macaques. Nat Commun 11:4400

Desautels JD, Moshirfar M, Martheswaran T, Shmunes KM, Ronquillo YC (2020) Risks posed to corneal transplant recipients by COVID-19-affected donors. Ophthalmol Ther 6:371-379

Dong M, Zhang J, Ma X, et al (2020) ACE2, TMPRSS2 distribution and extrapulmonary organ injury in patients with COVID-19. Biomem Pharmacother 131:110678

Fang Y, Zhang H, Xie J et al (2020) Sensitivity of chest CT for COVID-19: comparison to RT-PCR. Radiology 296:E115-E117

Food \& Drug Administration (FDA), Center for Biologics Evaluation and Research (CBER) (2020) Updated Information for Human Cell, Tissue, or Cellular or Tissue-based Product (HCT/P) Establishments Regarding the COVID19 Pandemic. U.S. Food \& Drug Administration (FDA). https://www.fda.gov/vaccines-blood-biologics/safetyavailability-biologics/updated-information-human-celltissue-or-cellular-or-tissue-based-product-hctpestablishments. Accessed 8 Oct 2020

Fu J, Zhou B, Zhang L et al (2020) Expressions and significances of the angiotensin-converting enzyme 2 gene, the receptor of SARS-CoV-2 for COVID-19. Mol Biol Rep 47:4383-4392

Hamashima K, Gautam P, Lau KA, et al (2020) Potential modes of COVID-19 transmission from human eye revealed by single-cell atlas. bioRxiv 2020.05.09.085613. https://doi. org/10.1101/2020.05.09.085613v3

Hamming I, Timens W, Bulthuis MLC, Lely AT, Navis GJ, van Goor H (2004) Tissue distribution of ACE2 protein, the functional receptor for SARS coronavirus. A first step in understanding SARS pathogenesis. J Pathol 203:631-637

He J-L, Luo L, Luo Z-D et al (2020a) Diagnostic performance between CT and initial real-time RT-PCR for clinically suspected 2019 coronavirus disease (COVID-19) patients outside Wuhan, China. Respir Med 168:105980

He X, Lau EHY, Wu P et al (2020b) Temporal dynamics in viral shedding and transmissibility of COVID-19. Nat Med 26:672-675

Hikmet F, Méar L, Edvinsson Å, Micke P, Uhlén M, Lindskog C (2020) The protein expression profile of ACE2 in human tissues. Mol Syst Biol 16:e9610

Ho D, Low R, Tong L et al (2020) COVID-19 and the ocular surface: a review of transmission and manifestations. Ocular Immunol Manifestat 28:725-734

Hui KPY, Cheung M-C, Perera RAPM et al (2020) Tropism, replication competence, and innate immune responses of the coronavirus SARS-CoV-2 in human respiratory tract and conjunctiva: an analysis in ex-vivo and in-vitro cultures. Lancet Respir Med 8:687-695

LeBlanc JJ, Heinstein C, MacDonald J, Pettipas J, Hatchette TF, Patriquin G (2020) A combined oropharyngeal/nares swab is a suitable alternative to nasopharyngeal swabs for the detection of SARS-CoV-2. J Clin Virol 128:104442

Li M-Y, Li L, Zhang Y, Wang X-S (2020) Expression of the SARS-CoV-2 cell receptor gene ACE2 in a wide variety of human tissues. Infect Dis Poverty 9:45
Li X, Chan JF-W, Li KK-W et al (2021) Detection of SARS$\mathrm{CoV}-2$ in conjunctival secretions from patients without ocular symptoms. N Engl J Med 382:723-733

Long C, Xu H, Shen Q et al (2020) Diagnosis of the coronavirus disease (COVID-19): rRT-PCR or CT? Eur J Radiol 126:108961

Lu CW, Liu XF, Jia ZF (2020) 2019-nCoV transmission through the ocular surface must not be ignored. Lancet 22:e39

Ma D, Chen CB, Jhanji V et al (2020) Expression of SARSCoV-2 receptor ACE2 and TMPRSS2 in human primary conjunctival and pterygium cell lines and in mouse cornea. Eye (lond) 34(7):1212-1219

Makovoz B, Moeller R, Zebitz Eriksen A, tenOever BR, Blenkinsop TA (2020) SARS-CoV-2 infection of ocular cells from human adult donor eyes and hESC-derived eye organoids. https://doi.org/10.2139/ssrn.3650574

Mason RJ (2020) Pathogenesis of COVID-19 from a cell biology perspective. Eur Respir J 55(4)

Muus C, Luecken MD, Eraslan G et al (2021) Single-cell metaanalysis of SARS-CoV-2 entry genes across tissues and demographics. Nat Med 27(3):546-559

Ortiz ME, Thurman A, Pezzulo AA et al (2020) Heterogeneous expression of the SARS-Coronavirus-2 receptor ACE2 in the human respiratory tract. EBioMedicine 60:102976. https://doi.org/10.1016/j.ebiom.2020.102976

Public Health Agency of Canada (2020a) Epidemiological summary of COVID-19 cases in Canada https://healthinfobase.canada.ca/covid-19/epidemiological-summarycovid-19-cases.html\#a4 .Accessed 8 Oct 2020

Public Health Agency of Canada (2020b) Coronavirus disease 2019 (COVID-19): epidemiology update. https://healthinfobase.canada.ca/covid-19/epidemiological-summarycovid-19-cases.html. Accessed 8 Oct 2020

Qin L, Yang Y, Cao Q et al (2020) A predictive model and scoring system combining clinical and CT characteristics for the diagnosis of COVID-19. Eur Radiol 30:6797-6807. https://doi.org/10.1007/s00330-020-07022-1

Sarma P, Kaur H, Kaur H, et al (2020) Ocular manifestations and tear or conjunctival swab PCR positivity for 2019-nCoV in patients with COVID-19: a systematic review and meta-analysis. https://doi.org/10.2139/ssrn. 3566161

Seah IYJ, Anderson DE, Kang AEZ et al (2020) Assessing viral shedding and infectivity of tears in coronavirus disease 2019 (COVID-19) patients. Ophthalmology 127:977-979

Singh M, Bansal V, Feschotte C (2020) A single-cell RNA expression map of human coronavirus entry factors. Cell Rep 32:108175

Statistics Canada / Government of Canada 1995 Statistics Canada: Canada's national statistical agency. https://www. statcan.gc.ca/eng/start. Accessed 8 Oct 2020

Sungnak W, Huang N, Bécavin C et al (2020) SARS-CoV-2 entry factors are highly expressed in nasal epithelial cells together with innate immune genes. Nat Med 26:681-687

Tuite AR, Fisman DN, Greer AL (2020) Mathematical modelling of COVID-19 transmission and mitigation strategies in the population of Ontario, Canada. CMAJ 192:E497-505

van Kasteren PB, van der Veer B, van den Brink S et al (2020) Comparison of seven commercial RT-PCR diagnostic kits for COVID-19. J Clin Virol 128:104412 
Vlek ALM, Wesselius TS, Achterberg R, Thijsen SFT (2021) Combined throat/nasal swab sampling for SARS-CoV-2 is equivalent to nasopharyngeal sampling. Eur $\mathrm{J}$ Clin Microbiol Infect Dis. https://doi.org/10.1007/s10096-02003972-y40:193-195

World Health Organization (2020) Timeline of WHO's response to COVID-19. World Health Organization. https://www.who.int/news-room/detail/29-06-2020covidtimeline. Accessed Oct 72020

Wu P, Duan F, Luo C et al (2020) Characteristics of ocular findings of patients with coronavirus disease 2019 (COVID-19) in Hubei province, China. JAMA Ophthalmol 138:575-578

Xia J, Tong J, Liu M, Shen Y, Guo D (2020) Evaluation of coronavirus in tears and conjunctival secretions of patients with SARS-CoV-2 infection. J Med Virol 92:589-594

Zhang X, Chen X, Chen L et al (2020a) The evidence of SARSCoV-2 infection on ocular surface. Ocul Surf 18:360-362

Zhang Y, Wang C, Han M et al (2020b) Discrimination of false negative results in RT-PCR detection of SARS-CoV-2 RNAs in clinical specimens by using an internal reference. Virol Sin. https://doi.org/10.1007/s12250-020-00273-835: 758-767
Zhang Y, Wang C, Han M et al (2020c) Correction to: discrimination of false negative results in RT-PCR detection of SARS-CoV-2 RNAs in clinical specimens by using an internal reference. Virol Sin. https://doi.org/10.1007/ s12250-020-00293-435:885-886

Zhou Y, Zeng Y, Tong Y, Chen C (2020) Ophthalmologic evidence against the interpersonal transmission of 2019 novel coronavirus through conjunctiva. medRxiv 2020.02.11.20021956. https://doi.org/10.1101/2020.02.11. 20021956v1

Ziegler CGK, Allon SJ, Nyquist SK et al (2020) SARS-CoV-2 receptor ACE2 is an interferon-stimulated gene in human airway epithelial cells and is detected in specific cell subsets across tissues. Cell 181:1016-1035.e19

Zou X, Chen K, Zou J, Han P, Hao J, Han Z (2020) Single-cell RNA-seq data analysis on the receptor ACE2 expression reveals the potential risk of different human organs vulnerable to 2019-nCoV infection. Front Med 14:185-192

Publisher's Note Springer Nature remains neutral with regard to jurisdictional claims in published maps and institutional affiliations. 